\title{
ON SUPERQUADRATIC ELLIPTIC SYSTEMS
}

\author{
DJAIRO G. DE FIGUEIREDO AND PATRICIO L. FELMER
}

ABSTRACT. In this article we study the existence of solutions for the elliptic system

$$
\begin{gathered}
-\Delta u=\frac{\partial H}{\partial v}(u, v, x) \text { in } \Omega, \\
-\Delta v=\frac{\partial H}{\partial u}(u, v, x) \text { in } \Omega, \\
u=0, \quad v=0 \quad \text { on } \partial \Omega .
\end{gathered}
$$

where $\Omega$ is a bounded open subset of $\mathbb{R}^{N}$ with smooth boundary $\partial \Omega$, and the function $H: \mathbb{R}^{2} \times \bar{\Omega} \rightarrow \mathbb{R}$, is of class $C^{1}$. We assume the function $H$ has a superquadratic behavior that includes a Hamiltonian of the form

$$
H(u, v)=|u|^{\alpha}+|v|^{\beta} \quad \text { where } 1-\frac{2}{N}<\frac{1}{\alpha}+\frac{1}{\beta}<1 \text { with } \alpha>1, \beta>1 .
$$

We obtain existence of nontrivial solutions using a variational approach through a version of the Generalized Mountain Pass Theorem. Existence of positive solutions is also discussed.

\section{INTRODUCTION}

This paper is devoted to the study of existence of solutions for certain superquadratic elliptic systems of the form

$$
\begin{aligned}
-\Delta u & =\frac{\partial H}{\partial v}(u, v, x) \quad \text { in } \Omega, \\
-\Delta v & =\frac{\partial H}{\partial u}(u, v, x) \quad \text { in } \Omega, \\
u & =0, \quad v=0 \quad \text { on } \partial \Omega,
\end{aligned}
$$

where $\Omega$ is a bounded open subset of $\mathbb{R}^{N}$, with smooth boundary $\partial \Omega$, and the function $H: \mathbb{R}^{2} \times \bar{\Omega} \rightarrow \mathbb{R}$, which we call the Hamiltonian, is of class $C^{1}$.

For easy reference later on we call the above problem (ES). The term "superquadratic" used here comes from hypothesis (H2) on the Hamiltonian (see it in the sequel, as well as Remark 0.5 ). Such a condition actually takes into account the coupling of the system. It does not imply that both equations in (ES)

Received by the editors April 10, 1992.

1980 Mathematics Subject Classification (1985 Revision). Primary 35J50, 58E99.

Key words and phrases. Elliptic systems, positive solutions, variational method.

The first author was partially supported by CNPq.

The second author was supported by FONDECYT under grant No 1212-91. 
are superquadratic, but it is implied by that, which follows from a condition like

$$
\text { (S) } \frac{\partial H}{\partial u}(u, v, x) \cdot u+\frac{\partial H}{\partial v}(u, v, x) \cdot v \geq \mu H(u, v, x)>0
$$

for all $(u, v) \in \mathbb{R}^{2} \backslash\{(0,0)\}, x \in \bar{\Omega}$, and $\mu>2$. Observe that (S) is a special case of (H2). We should mention that Benci and Rabinowitz [2] have already considered a special case of (ES) when both equations are superlinear, namely

$$
-\Delta w=\left(w^{2}+z^{2}\right)^{(s-1) / 2} w, \quad-\Delta z=-\left(w^{2}+z^{2}\right)^{(s-1) / 2} z,
$$

where $s>1$. Such a system is of the form

$$
\begin{aligned}
-\Delta w & =\frac{\partial H}{\partial w}(w, z, x), \\
-\Delta z & =-\frac{\partial H}{\partial z}(w, z, x)
\end{aligned}
$$

which is equivalent to our system (ES). In studying (0.5) and (0.6) Benci and Rabinowitz used the Generalized Mountain Pass Theorem in its infinite dimensional setting.

Recently in [4] Clement, de Figueiredo and Mitidieri studied a case of (ES) including Hamiltonians satisfying

$$
H(u, v)=|u|^{\alpha}+|v|^{\beta}
$$

where $\frac{1}{\alpha}+\frac{1}{\beta}<1, \alpha>1$ and $\beta>1$, but where the case $\alpha<2$ is allowed. Thus not satisfying (S). In [4] a priori estimates for positive solutions were obtained and then degree theory arguments were used to prove the existence of positive solutions for (ES). In another work, Clement, de Figueiredo and Mitidieri [5] have considered some classes of superlinear elliptic systems with growth that allows the use of inequalities of the Hardy-Sobolev type, and have obtained a priori bounds for positive solutions.

Some other class of superlinear elliptic systems were also considered by Souto [13]. He used the techniques introduced by Gidas and Spruck [8] in order to obtain a priori bounds for positive solutions, and by degree theory existence of positive solutions was established. Sublinear systems with a Hamiltonian form were discussed in Costa and Magalhães [3].

In this paper we consider superquadratic Hamiltonians using a variational approach. This allows us to extend the results in [2 and 4]. This kind of Hamiltonian was studied recently by Felmer [6] in the context of Hamiltonian systems.

Next we describe our results in a more precise way. On the Hamiltonian $H$ we will consider the following hypotheses:

(H0) $H: \mathbb{R}^{2} \times \bar{\Omega} \rightarrow \mathbb{R}$ is of class $C^{1}$.

(H1) $H(u, v, x) \geq 0$ for all $(u, v, x) \in \mathbb{R}^{2} \times \bar{\Omega}$.

Let us consider real constants $p \geq \alpha>p-1>0$ and $q \geq \beta>q-1>0$ 
such that

$$
\begin{aligned}
& \frac{1}{\alpha}+\frac{1}{\beta}<1, \\
& \left\{2-\left(\frac{1}{p}+\frac{1}{q}\right)\right\} \max \left\{\frac{p}{\alpha}, \frac{q}{\beta}\right\}<1+\frac{2}{N}, \\
& \frac{p-1}{p} \frac{q}{\beta}<1 \text { and } \frac{q-1}{q} \frac{p}{\alpha}<1 .
\end{aligned}
$$

In this paper we will always assume $N \geq 3$. If $N=2$ or $N=1$ less restrictive assumptions can be made.

Furthermore, in case $N \geq 5$ we also impose

$$
\left(1-\frac{1}{p}\right) \max \left\{\frac{p}{\alpha}, \frac{q}{\beta}\right\}<\frac{N+4}{2 N} \text { and }\left(1-\frac{1}{q}\right) \max \left\{\frac{p}{\alpha}, \frac{q}{\beta}\right\}<\frac{N+4}{2 N} \text {. }
$$

With these constants $\alpha, \beta, p, q$ satisfying the above conditions (i)-(iv) we now state the further hypotheses on the Hamiltonian $H$ :

(H2) There exists $R>0$ such that

$$
\frac{1}{\alpha} \frac{\partial H}{\partial u}(u, v, x) \cdot u+\frac{1}{\beta} \frac{\partial H}{\partial v}(u, v, x) \cdot v \geq H(u, v, x)>0
$$

for all $(u, v) \in \mathbb{R}^{2},|(u, v)| \geq R$ and $x \in \bar{\Omega}$.

(H3) There exists $r>0$ and $a_{1}>0$ such that

$$
H(u, v, x) \leq a_{1}\left(|u|^{\alpha}+|v|^{\beta}\right) \quad \text { if }|(u, v)| \leq r
$$

and

(H4) There exists $a_{2}>0$ such that

$$
\begin{aligned}
& \left|\frac{\partial H}{\partial u}(u, v, x)\right| \leq a_{2}\left(|u|^{p-1}+|v|^{(p-1) q / p}+1\right), \\
& \left|\frac{\partial H}{\partial v}(u, v, x)\right| \leq a_{2}\left(|v|^{q-1}+|u|^{(q-1) p / q}+1\right) .
\end{aligned}
$$

Our first existence results consider the concept of strong solution. In our situation we have

Definition 0.1. We say that $(u, v)$ is a strong solution of (ES) if

$$
u \in W^{2, p /(p-1)}(\Omega) \cap W_{0}^{1, p /(p-1)}(\Omega), \quad v \in W^{2, q /(q-1)}(\Omega) \cap W_{0}^{1, q /(q-1)}(\Omega)
$$

and $(u, v)$ satisfies $(0.1)$ and (0.2) a.e. in $\Omega$.

We will prove the following results.

Theorem 0.1. If $H$ satisfies $(\mathrm{H} 0)-(\mathrm{H} 4)$ then system $(\mathrm{ES})$ possesses at least one strong solution.

Theorem 0.2. If $H$ satisfies $(\mathrm{H} 0)-(\mathrm{H} 4)$ and $H$ is independent of $x \in \bar{\Omega}$ then (ES) possesses at least one nontrivial strong solution.

Remark 0.1 . When we assume more regularity on the Hamiltonian $H$, using standard arguments, it can be proved that strong solutions are indeed classical solutions. See [9]. In this direction a hypothesis to consider is 
$\left(\mathrm{HO}^{\prime}\right) H: \mathbb{R}^{2} \times \bar{\Omega} \rightarrow \mathbb{R}$ is of class $C^{1, \varepsilon}$.

When some more assumptions are considered in $H$ we also obtain a result on existence of positive classical solutions.

Theorem 0.3. If $H$ satisfies $\left(\mathrm{H}^{\prime}\right),(\mathrm{H} 1)-(\mathrm{H} 4)$ and also

(H5) $\partial H(u, v, x) / \partial u \geq 0, \partial H(u, v, x) / \partial v \geq 0$ for all $(u, v) \in \mathbb{R}^{2}, u \geq$ $0, v \geq 0, x \in \bar{\Omega}$,

(H6) $\partial H(u, v, x) / \partial u=0, \partial H(u, v, x) / \partial v=0$ if $u=0$ or $v=0$.

Then (ES) possesses at least one positive solution $(u, v)$ with $u(x)>0, v(x)>$ 0 if $x \in \Omega$.

Next we make some remarks about the hypotheses we considered.

Remark 0.2. Since $\frac{p}{\alpha} \geq 1$ and $\frac{q}{\beta} \geq 1$ we have from (ii) that

$$
1>\frac{1}{\alpha}+\frac{1}{\beta} \geq \frac{1}{p}+\frac{1}{q}>1-\frac{2}{N} \text {. }
$$

The first inequality above expresses the superquadratic character of the Hamiltonian $H$, as said before. The last inequality expresses the subcritical character of the system, as already pointed out in [4]. It seems however that these minimal assumptions do not suffice. Some further control on the constants $\alpha, \beta, p, q$ as expressed in (ii) and (iii) seems to be necessary.

Remark 0.3. Besides the critical curve $\frac{1}{p}+\frac{1}{q}>1-\frac{2}{N}$, in case $N \geq 5$ we have two extra critical lines as given by (iv).

Remark 0.4. If $\alpha=p, \beta=q$ then (i) and (ii) simply express

$$
1>\frac{1}{\alpha}+\frac{1}{\beta}>1-\frac{2}{N}
$$

and (iii) trivializes. Condition (iv) becomes

$$
\frac{1}{p}>\frac{N-4}{2 N}, \quad \frac{1}{q}>\frac{N-4}{2 N},
$$

see [4].

Remark 0.5 . It follows from $(\mathrm{H} 2)$ that there are constants $c_{1}>0$ and $c_{2}>0$ such that

$$
H(u, v, x) \geq c_{1}\left(|u|^{\alpha}+|v|^{\beta}\right)-c_{2}
$$

For a proof see [6].

Our Theorem 0.3 generalizes Theorem 3.1 in [4]. Let $f, g: \mathbb{R} \rightarrow \mathbb{R}$ functions satisfying the hypothesis of Theorem 3.1 in [4]. Then we can define a Hamiltonian

$$
H(u, v)=G(u)+F(v)
$$


with $G(u)=\int_{0}^{u} g(s) d s$ and $F(v)=\int_{0}^{v} f(s) d s$. This Hamiltonian satisfies our hypotheses. In particular it satisfies (H5) and (H6).

\section{The Variational formulation of (ES)}

In this section we set up the functional analytic framework needed to study problem (ES) from the variational point of view. We also give the variational formulation of (ES).

We shall work with spaces $E^{s}$, which are obtained as the domains of fractional powers of the operator

$$
-\Delta: H^{1}(\Omega) \cap H_{0}^{1}(\Omega) \subset L^{2}(\Omega) \rightarrow L^{2}(\Omega)
$$

where $\Delta$ denotes the Laplacian and $H^{2}(\Omega), H_{0}^{1}(\Omega)$ are the usual Sobolev spaces. Namely $E^{s}=D\left((-\Delta)^{s / 2}\right)$ for $0 \leq s \leq 2$, and the corresponding operator is denoted by $A^{s}$

$$
A^{s}: E^{s} \rightarrow L^{2}(\Omega) \text {. }
$$

The spaces $E^{s}$ are Hilbert spaces with inner product

$$
(u, v)_{E^{s}}=\int_{\Omega} A^{s} u A^{s} v d x
$$

and associated norm

$$
\|u\|_{E^{s}}^{2}=\int_{\Omega}\left|A^{s} u\right|^{2} d x .
$$

It is known that these spaces are indeed Sobolev spaces of fractional power as defined by interpolation, see Lions and Magenes [10]. In particular we have

$$
\begin{gathered}
E^{s}=H^{s}(\Omega) \quad \text { if } 0 \leq s<\frac{1}{2}, \quad E^{1 / 2} \subset H^{1 / 2}(\Omega), \\
E^{s}=\left\{u \in H^{s}(\Omega) \mid u=0 \text { on } \partial \Omega\right\} \text { if } \frac{1}{2}<s \leq 2, s \neq \frac{3}{2}, \quad \text { and } \\
E^{3 / 2} \subset\left\{u \in H^{3 / 2}(\Omega) \mid u=0 \text { on } \partial \Omega\right\} .
\end{gathered}
$$

See also the results in Fujiwara [7].

If we consider a basis of $L^{2}(\Omega)$ constituted by eigenfunctions $\left\{\phi_{n}\right\}$ of

$$
-\Delta \phi=\lambda \phi \quad \text { in } \Omega, \quad \phi=0 \text { on } \partial \Omega
$$

with associated eigenvalues $\left\{\lambda_{n}\right\}$, then we have the following characterization of $E^{s}$ and $A^{s}$. If $u \in L^{2}(\Omega)$ we write $u=\sum_{n=1}^{\infty} a_{n} \phi_{n}$ its Fourier series with respect to the basis $\left\{\phi_{n}\right\}$. Then

$$
E^{s}=\left\{\left.u \in L^{2}(\Omega)\left|\sum_{n=1}^{\infty} \lambda_{n}^{s}\right| a_{n}\right|^{2}<\infty\right\}
$$

and

$$
A^{s} u=\sum_{n=1}^{\infty} \lambda_{n}^{s / 2} a_{n} \phi_{n}
$$

for all $u \in E^{s}$. Now it is easy to see Poincare's inequality for the operator $A^{s}$

$$
\left\|A^{s} u\right\|_{L^{2}(\Omega)} \geq \lambda_{1}^{s / 2}\|u\|_{L^{2}(\Omega)} \quad \forall u \in E^{s}
$$

where $\lambda_{1}$ is the first eigenvalue of $-\Delta$.

The fractional order spaces have the following important embedding property. 
Theorem 1.1. Given $s>0$ and $\sigma \geq 1$ so that $\frac{1}{\sigma} \geq \frac{1}{2}-\frac{2}{N}$ then the inclusion map $i: E^{s} \rightarrow L^{\sigma}(\Omega)$ is well defined and bounded. If above we have strict inequality then the inclusion is compact.

The proof of this theorem is based on interpolation and the Kondrachov Theorem. See the article of Persson [11] for a proof.

With these preliminaries about the Laplacian and the spaces we can now define the functional associated to (ES). Let us consider first the quadratic part. For numbers $s>0$ and $t>0$ with $s+t=2$ we define the Hilbert space $E=E^{s} \times E^{t}$ and the bilinear form $B: E \times E \rightarrow \mathbb{R}$ by the formula

$$
B((u, v),(\phi, \psi))=\int_{\Omega} A^{s} u A^{t} \psi+A^{s} \phi A^{t} v d x .
$$

Using the Cauchy Schwarz inequality and (1.2) it is easy to see that $B$ is continuous. We also see that $B$ is symmetric. Then $B$ induces a selfadjoint bounded linear operator $L: E \rightarrow E$ so that

$$
B(z, \eta)=(L z, \eta)_{E}
$$

for all $z, \eta \in E$. Here and in what follows $(\cdot, \cdot)_{E}$ denotes the inner product in $E$ induced by $(\cdot, \cdot)_{E^{s}}$ and $(\cdot, \cdot)_{E^{t}}$ on the product space $E$ in the usual way. We can also define the quadratic form $Q: E \rightarrow \mathbb{R}$ associated to $B$ and $L$ as

$$
Q(z)=\frac{1}{2}(L z, z)_{E}=\int_{\Omega} A^{s} u A^{t} v d x
$$

for all $z=(u, v) \in E$. A more explicit formula for $L$ will be needed in the future.

Proposition 1.1. The operator $L$ defined above can be written as

$$
L(u, v)=\left(\left(A^{s}\right)^{-1} A^{t} v,\left(A^{t}\right)^{-1} A^{s} u\right) .
$$

Proof. If $z=(u, v), \eta=(\phi, \psi) \in E$ and we write $L(z)=(w, y)$ then we have

$$
(L(z), \eta)_{E}=((w, y),(\phi, \psi))_{E}=\int_{\Omega} A^{s} w A^{s} \phi+A^{t} y A^{t} \psi d x .
$$

On the other hand, from (1.6) we have

$$
(L(z), \eta)_{E}=\int_{\Omega} A^{s} u A^{t} \psi+A^{s} \phi A^{t} v d x .
$$

Taking $\psi=0$ in (1.10) and (1.11) we obtain

$$
\int_{\Omega} A^{s} \phi\left(A^{t} v-A^{s} w\right) d x=0 \quad \forall \phi \in E^{s} .
$$

Using that $A^{s}$ is an isomorphism onto $L^{2}(\Omega)$ we conclude the equality $A^{t} v-$ $A^{s} w=0$ from where $w=\left(A^{s}\right)^{-1} A^{t} v$.

If we take $\phi=0$ in (1.10) and (1.11) we obtain in a similar way $y=$ $\left(A^{t}\right)^{-1} A^{s} u$.

In what follows we write $A^{-s}=\left(A^{s}\right)^{-1}$. Next we consider the eigenvalue problem

$$
L z=\lambda z \quad \text { in } E .
$$


Using Proposition 1.1 we can write (1.13) in equivalent form as

$$
\begin{aligned}
& A^{-s} A^{t} v=\lambda u, \\
& A^{-t} A^{s} u=\lambda v,
\end{aligned}
$$

where $z=(u, v)$. Since the operators $A^{s}$ and $A^{t}$ are isomorphisms $\lambda$ cannot be zero. Then isolating $u$ in (1.14) and substituting in (1.15) yields

$$
v=\lambda^{2} v
$$

whence it follows that $\lambda=1$ or $\lambda=-1$. The associated eigenvectors are

$$
\text { for } \lambda=1, \quad\left(u, A^{-t} A^{s} u\right) \quad \forall u \in E^{s}
$$

and

$$
\text { for } \lambda=-1, \quad\left(u,-A^{-t} A^{s} u\right) \quad \forall u \in E^{s} .
$$

We can define the eigenspaces

$$
E^{+}=\left\{\left(u, A^{-t} A^{s} u\right) \mid u \in E^{s}\right\}
$$

and

$$
E^{-}=\left\{\left(u,-A^{-t} A^{s} u\right) \mid u \in E^{s}\right\}
$$

which give a natural splitting $E=E^{+} \oplus E^{-}$. The spaces $E^{+}$and $E^{-}$are orthogonal with respect to the bilinear form $B$, that is

$$
B\left(z^{+}, z^{-}\right)=0 \quad \forall z^{+} \in E^{+}, z^{-} \in E^{-} .
$$

We also find that

$$
\frac{1}{2}\|z\|_{E}^{2}=Q\left(z^{+}\right)-Q\left(z^{-}\right)
$$

where $z=z^{+}+z^{-}, z_{ \pm} \in E^{ \pm}$.

Next we define the functional associated to the Hamiltonian. Using the growth hypothesis (H4) and integrating we obtain

$$
H(u, v, x) \leq|H(0, v, x)|+a_{2}\left(|u|^{p-1}+|v|^{(p-1) q / p}+1\right)|u|
$$

and

$$
|H(0, v, x)| \leq|H(0,0, x)|+a_{2}\left(|v|^{q}+|v|\right) .
$$

Young's inequality implies

$$
|v|^{(p-1) q / p}|u| \leq \frac{|u|^{p}}{p}+\frac{(p-1)}{p}|v|^{q} .
$$

From (1.21)-(1.23) we obtain

$$
|H(u, v, x)| \leq c_{2}\left(|u|^{p}+|v|^{q}\right)+c_{3}
$$

for certain constants $c_{2}$ and $c_{3}$. Now we will choose the numbers $s$ and $t$ defining the orders of the Sobolev spaces involved. From inequality (ii) in the Introduction we see the existence of $s, t \in \mathbb{R}, s+t=2$ such that

$$
\left(1-\frac{1}{p}\right) \max \left\{\frac{p}{\alpha}, \frac{q}{\beta}\right\}<\frac{1}{2}+\frac{s}{N}
$$


and

$$
\left(1-\frac{1}{q}\right) \max \left\{\frac{p}{\alpha}, \frac{q}{\beta}\right\}<\frac{1}{2}+\frac{t}{N} \text {. }
$$

Using (iii) and (iv) if $N \geq 5$, we can choose $s>0$ and $t>0$. From the fact that $\frac{p}{\alpha} \geq 1$ and $\frac{q}{\beta} \geq 1$ we find, following from (1.25) and (1.26) that

$$
\frac{1}{p}>\frac{1}{2}-\frac{s}{N} \text { and } \quad \frac{1}{q}>\frac{1}{2}-\frac{t}{N} \text {. }
$$

These last inequalities and Theorem 1.1 give the compact inclusions $E^{s} \rightarrow L^{p}(\Omega), E^{t} \rightarrow L^{q}(\Omega)$. Then using (1.24) we can define the functional $\mathscr{H}: E \rightarrow \mathbb{R}$ as

$$
\mathscr{H}(u, v)=\int_{\Omega} H(u(x), v(x), x) d x .
$$

Proposition 1.2. The functional $\mathscr{H}$ defined above is of class $C^{1}$ and its derivative is given by

$$
\mathscr{H}^{\prime}(u, v)(\phi, \psi)=\int_{\Omega} \frac{\partial H}{\partial u}(u, v, x) \phi+\frac{\partial H}{\partial v}(u, v, x) \psi d x
$$

for all $(u, v),(\phi, \psi) \in E$. Moreover $\mathscr{H}^{\prime}: E \rightarrow E$ is a compact operator.

Proof. The expression given at the right-hand side of (1.29) is well defined. In fact, from the growth hypothesis (H4) we have

$$
\int_{\Omega}\left|\frac{\partial H}{\partial u}(u, v, x) \phi\right| d x \leq a_{2} \int_{\Omega}\left(|u|^{p-1}+|v|^{(p-1) q / p}+1\right)|\phi| d x .
$$

Using Hölder inequality and embeddings

$$
\int_{\Omega}\left|\frac{\partial H}{\partial u}(u, v, x) \phi\right| d x \leq a_{2}\left(\|u\|_{E^{s}}^{p-1}+\|v\|_{E^{t}}^{(p-1) q / p}+1\right)\|\phi\|_{E^{s}}
$$

In a similar way we obtain an inequality for the derivative with respect to $v$. Thus $\mathscr{H}^{\prime}(u, v)$ is well defined and bounded in $E$.

Next, usual arguments give that $\mathscr{H}$ is Fréchet differentiable, $\mathscr{H}^{\prime}$ is continuous and, as a consequence of the Sobolev embeddings, $\mathscr{H}^{\prime}$ is also compact. See [12] for example.

Now we can define a functional $\Phi: E \rightarrow \mathbb{R}$ as

$$
\Phi(z)=Q(z)-\mathscr{H}(z) \quad \forall z \in E .
$$

$\Phi$ is a functional of class $C^{1}$ and by previous considerations it has the structure needed to apply minimax techniques. See (3.6).

Definition 1.1. We say that $z=(u, v) \in E=E^{s} \times E^{t}$ is an $(s, t)$-weak solution of (ES) if $z$ is a critical point of $\Phi$. In other words, if for all $\eta=(\phi, \psi) \in$ $E^{s} \times E^{t}$ we have

$$
\int_{\Omega} A^{s} u A^{t} \psi+A^{s} \phi A^{t} v-\frac{\partial H}{\partial u}(u, v, x) \phi-\frac{\partial H}{\partial v}(u, v, x) \psi d x=0 .
$$

The next theorem gives a regularity result for $(s, t)$-weak solutions. 
Theorem 1.2. If $(u, v) \in E^{s} \times E^{t}$ is an $(s, t)$-weak solution of (ES) then $u \in$ $W^{2, p /(p-1)}(\Omega) \cap W_{0}^{1, p /(p-1)}(\Omega), v \in W^{2, q /(q-1)}(\Omega) \cap W_{0}^{1, q /(q-1)}(\Omega)$ and

$$
\begin{aligned}
-\Delta u & =\frac{\partial H}{\partial v}(u, v, x), \\
-\Delta v & =\frac{\partial H}{\partial u}(u, v, x)
\end{aligned}
$$

a.e. in $\Omega$. In other words $(u, v)$ is a strong solution of $(\mathrm{ES})$.

Proof. Let us consider $\psi=0$ in (1.33), then

$$
\int_{\Omega} A^{t} v A^{s} \phi d x=\int_{\Omega} \frac{\partial H}{\partial u}(u, v, x) \phi d x
$$

for all $\phi \in E^{s}$. If $\phi \in H^{2}(\Omega) \cap H_{0}^{1}(\Omega)$ then we have

$$
\int_{\Omega} A^{t} v A^{s} \phi d x=\int_{\Omega} v A^{2} \phi d x=-\int_{\Omega} v \Delta \phi d x .
$$

On the other hand, using estimates following from (H4) we find

$$
\frac{\partial H}{\partial u}(u(x), v(x), x) \in L^{p /(p-1)}(\Omega),
$$

and then from basic elliptic theory there exists one function $w \in W^{2, p /(p-1)}(\Omega)$ $\cap W_{0}^{1, p /(p-1)}(\Omega)$ such that

$$
-\Delta w=\frac{\partial H}{\partial u}(u(x), v(x), x),
$$

see Gilbarg and Trudinger [9]. Following from (1.27)

$$
\frac{1}{2}>\frac{p-1}{p}-\frac{s}{N}>\frac{p-1}{p}-\frac{2}{N}
$$

so that from the embedding theorem of Sobolev $w \in L^{2}(\Omega)$ (see [1]). Thus we have

$$
\int_{\Omega} \frac{\partial H}{\partial u}(u, v, x) \phi d x=-\int_{\Omega} \Delta w \phi d x=-\int_{\Omega} w \Delta \phi d x
$$

for all $\phi \in H^{2}(\Omega) \cap H_{0}^{1}(\Omega)$. Next, from (1.37) and (1.39) we obtain

$$
\int_{\Omega}(v-w) \Delta \phi d x \quad \forall \phi \in H^{2}(\Omega) \cap H_{0}^{1}(\Omega)
$$

from where it follows that $v=w$. We have obtained that $v \in W^{2, p /(p-1)}(\Omega) \cap$ $W_{0}^{1, p /(p-1)}(\Omega)$. Finally, since $v=w$ we conclude that $v$ satisfies (1.35).

We can do the same reasoning for $u$.

\section{Palais Smale and geometric conditions}

In this section we further study the functional $\Phi$. We prove the Palais Smale condition for $\Phi$ and then obtain the geometric situation of the Generalized Mountain Pass Theorem. 
Proposition 2.1. $\Phi$ satisfies the Palais Smale condition.

Proof. Let $\left\{z_{n}\right\}$ be a sequence in $E$ so that

$$
\left|\Phi\left(z_{n}\right)\right| \leq c \text { and } \Phi^{\prime}\left(z_{n}\right) \rightarrow 0, \quad \text { as } n \rightarrow \infty .
$$

We prove first that (2.1) implies that $\left\{z_{n}\right\}$ is bounded. From (2.1) there is a sequence $\left\{\varepsilon_{n}\right\}$ converging to 0 so that

$$
\left|\Phi^{\prime}\left(z_{n}\right) \eta\right| \leq \varepsilon_{n}\|\eta\|_{E} \quad \forall \eta \in E .
$$

Taking

$$
\eta_{n}=\frac{\alpha \beta}{\alpha+\beta}\left(\frac{1}{\alpha} u_{n}, \frac{1}{\beta} v_{n}\right), \quad \text { where } z_{n}=\left(u_{n}, v_{n}\right),
$$

and using (2.1) we find

$$
\begin{aligned}
c+\varepsilon_{n}\left\|\eta_{n}\right\|_{E} \geq & \Phi\left(z_{n}\right)-\Phi^{\prime}\left(z_{n}\right) \eta_{n}=\frac{\alpha \beta}{\alpha+\beta} \int_{\Omega} \frac{1}{\alpha} \frac{\partial H}{\partial u}\left(u_{n}, v_{n}, x\right) u_{n} \\
& +\frac{1}{\beta} \frac{\partial H}{\partial v}\left(u_{n}, v_{n}, x\right) v_{n}-H\left(u_{n}, v_{n}, x\right) d x \\
& +\left(\frac{\alpha \beta}{\alpha+\beta}-1\right) \int_{\Omega} H\left(u_{n}, v_{n}, x\right) d x .
\end{aligned}
$$

Using (H2) we find a constant $c_{1}$ so that

$$
c_{1}\left(1+\left\|z_{n}\right\|_{E}\right) \geq \int_{\Omega} H\left(u_{n}, v_{n}, x\right) d x .
$$

and then, using (0.8) we obtain a constant $c_{3}$ such that

$$
\int_{\Omega}\left|u_{n}\right|^{\alpha}+\left|v_{n}\right|^{\beta} d x \leq c_{2}\left(1+\left\|u_{n}\right\|_{E^{s}}+\left\|v_{n}\right\|_{E^{t}}\right) .
$$

Next, let us consider $\eta=(\phi, 0)$ with $\phi \in E^{s}$. Then from (2.2)

$$
\left|\int_{\Omega} A^{s} \phi A^{t} v_{n} d x\right| \leq \int_{\Omega}\left|\frac{\partial H}{\partial u}\left(u_{n}, v_{n}, x\right) \phi\right| d x+\varepsilon_{n}\|\phi\|_{E^{s}} .
$$

We estimate these terms next. From (1.25) and Theorem 1.1 we find

$$
\|\phi\|_{L^{\alpha /(\alpha-p+1)}(\Omega)} \leq c_{1}\|\phi\|_{E^{s}}
$$

then, using Hölder inequality with $a=\alpha /(p-1), b=\alpha /(\alpha-p+1)$ we obtain

$$
\int_{\Omega}\left|u_{n}\right|^{p-1}|\phi| d x \leq c_{1}\left\|u_{n}\right\|_{L^{\alpha}(\Omega)}^{p-1}\|\phi\|_{E^{s}}
$$

From (iii) in the Introduction and (1.25) we have

$$
1-\frac{p-1}{p} \frac{q}{\beta}>\frac{1}{2}-\frac{s}{N}
$$

and then Theorem 1.1 implies the embedding of $E^{s}$ into $L^{\beta p /(\beta p-(p-1) q)}(\Omega)$. This fact together with Hölder inequality with $a=\beta p /(p-1) q$ and $b=$ $\beta p /(\beta p-(p-1) q)$ yields

$$
\int_{\Omega}\left|v_{n}\right|^{(p-1) q / p}|\phi| d x \leq c_{2}\left\|v_{n}\right\|_{L^{\beta}(\Omega)}^{(p-1) q / p}\|\phi\|_{E^{s}}
$$


Finally, again using Theorem 1.1

$$
\int_{\Omega}|\phi| d x \leq c_{3}\|\phi\|_{E^{s}}
$$

Putting together (H4), (2.6), (2.8)-(2.10) we obtain

$$
\left|\int_{\Omega} A^{s} \phi A^{t} v_{n} d x\right| \leq c_{4}\left(\left\|u_{n}\right\|_{L^{\alpha}(\Omega)}^{p-1}+\left\|v_{n}\right\|_{L^{\beta}(\Omega)}^{(p-1) q / p}+1\right)\|\phi\|_{E^{s}}
$$

for all $\phi \in E^{s}$, from where it follows

$$
\left\|v_{n}\right\|_{E^{t}} \leq c_{4}\left(\left\|u_{n}\right\|_{L^{\alpha}(\Omega)}^{p-1}+\left\|v_{n}\right\|_{L^{\beta}(\Omega)}^{(p-1) q / p}+1\right) .
$$

By an analogous reasoning

$$
\left\|u_{n}\right\|_{E^{s}} \leq c_{5}\left(\left\|v_{n}\right\|_{L^{\beta}(\Omega)}^{q-1}+\left\|u_{n}\right\|_{L^{\alpha}(\Omega)}^{(q-1) p / q}+1\right) .
$$

Replacing (2.12) and (2.13) into (2.5) we obtain

$$
\begin{aligned}
& \left\|u_{n}\right\|_{E^{s}}+\left\|v_{n}\right\|_{E^{t}} \\
& \quad \leq c_{6}\left(\left\|u_{n}\right\|_{E^{s}}^{(p-1) / \alpha}+\left\|v_{n}\right\|_{E^{t}}^{(p-1) q / p \beta}+\left\|u_{n}\right\|_{E^{s}}^{(q-1) p / q \alpha}+\left\|v_{n}\right\|_{E^{t}}^{(q-1) / \beta}+1\right) .
\end{aligned}
$$

Since the exponents in the right-hand side of $(2.14)$ are all less that 1 , by the basic assumptions we made on $\alpha, \beta, p$ and $q$, we find that the sequence $\left\{z_{n}\right\}$ is bounded in $E$.

From here on a usual argument based on the compactness of $\mathscr{H}^{\prime}$ and the invertibility of $L$ gives the existence of a subsequence of $\left\{z_{n}\right\}$ that converges in $E$.

We will consider now the study of the geometric characteristics of $\Phi$ leading to the Generalized Mountain Pass Theorem.

We will define subsets $S$ and $Q$ so that

(IS) There exists $\delta>0$ such that

$$
\Phi(z) \geq \delta \quad \forall z \in S .
$$

(IQ) $\Phi(z) \leq 0 \quad \forall z \in \partial Q$.

Where $\partial Q$ denotes the boundary of $Q$ relative to a certain subspace of $E$. It is shown also that $S$ and $\partial Q$ link in the sense of Benci and Rabinowitz.

For later reference we state a preliminary lemma giving the expressions of the projections over $E^{ \pm}$.

Lemma 2.1. The orthogonal projections $P^{ \pm}: E \rightarrow E^{ \pm}$are given by the formula

$$
P^{ \pm}(u, v)=\frac{1}{2}\left(u \pm A^{-s} A^{t} v, v \pm A^{-t} A^{s} u\right) .
$$

Proof. Direct from definitions.

Now we choose numbers $\mu>1, \nu>1$ such that

$$
\frac{1}{\alpha}<\frac{\mu}{\mu+\nu} \text { and } \frac{1}{\beta}<\frac{\nu}{\mu+\nu} .
$$

Proposition 2.2. There exist $\rho>0$ and $\delta>0$ such that if we define

$$
S=\left\{\left(\rho^{\mu-1} u, \rho^{\nu-1} v\right) \mid\|(u, v)\|=\rho,(u, v) \in E^{+}\right\}
$$

then (IS) is satisfied. 
Proof. Let $\tilde{z}=(u, v) \in E^{+}$and put $z=\left(\rho^{\mu-1} u, \rho^{\nu-1} v\right)$. Recall that $v=$ $A^{-t} A^{s} u$ and equivalently $u=A^{-s} A^{t} v$. We have

$$
Q(z)=\int_{\Omega} \rho^{\mu-1} A^{s} u \rho^{\nu-1} A^{t} v d x=\rho^{\mu+\nu-2} \int_{\Omega} A^{s} u A^{t} v d x
$$

Using (1.20) and (2.18) we get

$$
Q(z)=\frac{1}{2} \rho^{\mu+\nu-2}\|\tilde{z}\|_{E}^{2}
$$

On the other hand, from (H3) and (H4) we have

$$
H(u, v, x) \leq a_{1}\left(|u|^{\alpha}+|v|^{\beta}\right)+b_{1}\left(|u|^{p}+|v|^{q}\right)
$$

for some constants $a_{1}, b_{1}$, then

$$
\begin{aligned}
\mathscr{H}(z) \leq & a_{1}\left(\rho^{(\mu-1) \alpha} \int_{\Omega}|u|^{\alpha} d x+\rho^{(\nu-1) \beta} \int_{\Omega}|v|^{\beta} d x\right) \\
& +b_{1}\left(\rho^{(\mu-1) p} \int_{\Omega}|u|^{p} d x+\rho^{(\nu-1) q} \int_{\Omega}|v|^{q} d x\right) .
\end{aligned}
$$

Since $\alpha \leq p, \beta \leq q$, using Hölder inequality and Theorem 1.1 we find from (2.21) that

$$
\mathscr{H}(z) \leq b_{2}\left(\rho^{(\mu-1) \alpha}\|\tilde{z}\|_{E}^{\alpha}+\rho^{(\mu-1) p}\|\tilde{z}\|_{E}^{p}+\rho^{(\nu-1) \beta}\|\tilde{z}\|_{E}^{\beta}+\rho^{(\nu-1) q}\|\tilde{z}\|_{E}^{q}\right) .
$$

Putting (2.19) and (2.22) together and considering $\rho=\|\tilde{z}\|_{E}$ we have

$$
\Phi(z) \geq \frac{1}{2} \rho^{\mu+\nu}-b_{2}\left(\rho^{\mu \alpha}+\rho^{\mu p}+\rho^{\nu \beta}+\rho^{\nu q}\right) .
$$

Since $\alpha \leq p, \beta \leq q$, for $\rho$ small we obtain

$$
\Phi(z) \geq \frac{1}{2} \rho^{\mu+\nu}-2 b_{1}\left(\rho^{\mu \alpha}+\rho^{\nu \beta}\right)=\left(\frac{\rho^{\mu+\nu}}{4}-2 b_{1} \rho^{\mu \alpha}\right)+\left(\frac{\rho^{\mu+\nu}}{4}-2 b_{1} \rho^{\nu \beta}\right) .
$$

From our choice of $\mu$ and $\nu$ in (2.17) we see that if $\rho$ is small enough, there exists $\delta>0$ such that $\Phi(z) \geq \delta$ if $\|\tilde{z}\|_{E}=\rho$.

We next define the set $Q$. For some constants $\sigma>0$ and $M>0$ to be precised later on we define

$$
\begin{aligned}
Q=\left\{\tau\left(\sigma^{\mu-1} u_{+}, \sigma^{\nu-1} v_{+}\right)+\left(\sigma^{\mu-1} u, \sigma^{\nu-1} v\right) \mid\right. \\
\left.0 \leq \tau \leq \sigma, 0 \leq\|(u, v)\|_{E} \leq M,(u, v) \in E^{-}\right\}
\end{aligned}
$$

where $z_{+}=\left(u_{+}, v_{+}\right) \in E^{+}$with $u_{+}$some fixed eigenvector of $-\Delta$. We note that $z_{+}$is an eigenvector of $L$ associated to a positive eigenvalue (i.e. to 1 ). We assume $\left\|z_{+}\right\|_{E}=1$. We denote by $\partial Q$ the boundary of $Q$ relative to the subspace

$$
\left\{\tau\left(\sigma^{\mu-1} u_{+}, \sigma^{\nu-1} v_{+}\right)+\left(\sigma^{\mu-1} u, \sigma^{\nu-1} v\right) \mid \tau \in \mathbb{R},(u, v) \in E^{-}\right\}
$$

Proposition 2.3. There are constants $\sigma>0$ and $M>0$ such that for $Q$ defined as above we have $\Phi(z) \leq 0 \quad \forall z \in \partial Q$.

Proof. For $\tau \in \mathbb{R}_{+},(u, v) \in E^{-}$we set

$$
z=\tau\left(\sigma^{\mu-1} u_{+}, \sigma^{\nu-1} v_{+}\right)+\left(\sigma^{\mu-1} u, \sigma^{\nu-1} v\right)
$$


By the definitions of $E^{+}$and $E^{-}$we have

$$
v_{+}=A^{-t} A^{s} u_{+} \text {and } v=-A^{-t} A^{s} u \text {, }
$$

and then evaluating $Q$ in $z$ we obtain

$$
\begin{aligned}
Q(z) & =\int_{\Omega}\left(\tau \sigma^{\mu-1} A^{s} u_{+}+\sigma^{\mu-1} A^{s} u\right)\left(\tau \sigma^{\nu-1} A^{s} u_{+}-\sigma^{\nu-1} A^{s} u\right) d x \\
& =\frac{1}{2} \sigma^{\mu+\nu-2}\left(\tau^{2}-\|(u, v)\|_{E}^{2}\right) .
\end{aligned}
$$

On the other hand, we have from Remark 0.5 that

$$
\int_{\Omega} H(z, x) d x \geq c_{1} \int_{\Omega}\left(\sigma^{\alpha(\mu-1)}\left|\tau u_{+}+u\right|^{\alpha}+\sigma^{\beta(\nu-1)}\left|\tau v_{+}+v\right|^{\beta}\right) d x-|\Omega| c_{2} .
$$

Each $u$ can be written as $u=\gamma u_{+}+\hat{u}$, where $\hat{u}$ is orthogonal to $u_{+}$in the $L^{2}(\Omega)$ sense, and $\gamma$ is some real number. Using Hölder's inequality we obtain

$$
\begin{aligned}
(\tau+\gamma) \int_{\Omega}\left|u^{+}\right|^{2} d x & =\int_{\Omega}\left(\tau u^{+}+u\right) u^{+} d x \\
& \leq\left\|\tau u^{+}+u\right\|_{L^{\alpha}(\Omega)}\left\|u^{+}\right\|_{L^{\alpha^{\prime}}(\Omega)}
\end{aligned}
$$

or

$$
\tau+\gamma \leq c\left\|\tau u^{+}+u\right\|_{L^{\alpha}(\Omega)} .
$$

Similarly, observing that $A^{-t} A^{s} u^{+}=\lambda_{k}^{-t+s} u^{+}$, where $\lambda_{k}$ is the eigenvalue of $\left(-\Delta, H_{0}^{1}(\Omega)\right)$ whose eigenfunction is $u^{+}$, we obtain

$$
\begin{aligned}
\lambda_{k}^{-t+s}(\tau-\gamma) \int_{\Omega}\left|u^{+}\right|^{2} d x & =\int_{\Omega}\left(\tau v^{+}+v\right) u^{+} d x \\
& \leq\left\|\tau v^{+}+v\right\|_{L^{\beta}(\Omega)}\left\|u^{+}\right\|_{L^{\beta^{\prime}}(\Omega)}
\end{aligned}
$$

or

$$
\tau-\gamma \leq c\left\|\tau v^{+}+v\right\|_{L^{\beta}(\Omega)}
$$

If $\gamma \geq 0$ it follows from (2.26), (2.27) and (2.29) that

$$
\Phi(z) \leq \frac{\sigma^{\mu+\nu-2}}{2} \tau^{2}-c_{6} \tau^{\alpha} \sigma^{\alpha(\mu-1)}+|\Omega| c_{2}
$$

And if $\gamma \leq 0$ it follows from (2.26), (2.27) and (2.31) that

$$
\Phi(z) \leq \frac{\sigma^{\mu+\nu-2}}{2} \tau^{2}-c_{6} \tau^{\beta} \sigma^{\beta(\nu-1)}+|\Omega| c_{2}
$$

By the choice of $\mu$ and $\nu$, taking $\tau=\sigma$ large we see in (2.32) and (2.33) that $\Phi(z) \leq 0$.

Now we choose $M$. If $\tau \in[0, \sigma]$, from (2.26) and (2.27) we have

$$
\Phi(z) \leq \frac{1}{2} \sigma^{\mu+\nu}-\frac{1}{2} \sigma^{\mu+\nu-2}\|(u, v)\|_{E}^{2}+|\Omega| c_{2}
$$

then taking $\|(u, v)\|=M$ and $M$ large enough we find $\Phi(z) \leq 0$. We finish the proof if we show that when $\tau=0$ then also $\Phi(z) \leq 0$. By hypothesis (H1) this is direct after the definition of $\Phi$ and (2.26). 


\section{THE MINIMAX THEOREM}

In this section we formulate a minimax theorem which is a version of Theorem 5.9 of [12] and it was proved in [6]. We describe this result and then we show how to use it in our situation. We consider a Hilbert space $E$ with inner product $\langle$,$\rangle and norm \|\cdot\|$. We assume that $E$ has a splitting $E=X \oplus Y$, where the subspace $X$ and $Y$ are not necessarily orthogonal and both of them can be infinite dimensional. Let $\Phi: E \rightarrow \mathbb{R}$ be a functional having the following structure

$$
\Phi(z)=\frac{1}{2}\langle L z, z\rangle+\mathscr{H}(z)
$$

with

(I1) $L: E \rightarrow E$ is a linear, bounded, selfadjoint operator,

(I2) $\mathscr{H}^{\prime}$ is compact.

There are two linear bounded, invertible operators $B_{1}, B_{2}: E \rightarrow E$ satisfying

(I3) If $\omega \in \mathbb{R}_{0}^{+}$then the linear operator

$$
\widehat{B}(\omega)=P_{X} B_{1}^{-1} \exp (\omega L) B_{2}: X \rightarrow X
$$

is invertible.

Here $P_{X}$ denotes the projection of $E$ onto $X$ induced by the splitting $E=$ $X \oplus Y$. Let $\rho>0$ and define

$$
S=\left\{B_{1} z \mid\|z\|=\rho, z \in Y\right\} .
$$

For $z_{+} \in Y, z_{+} \neq 0, \sigma>\rho /\left\|B_{1}^{-1} B_{2} z_{+}\right\|$and $M>\rho$, we define

$$
Q=\left\{B_{2}\left(\tau z_{+}+z\right) \mid 0 \leq \tau \leq \sigma,\|z\| \leq M, z \in X\right\} .
$$

Where $\partial Q$ denotes the boundary of $Q$ relative to the subspace

$$
\left\{B_{2}\left(\tau z_{+}+z\right) \mid \tau \in \mathbb{R}, z \in X\right\} .
$$

Then we have the following theorem on existence of critical points of $\Phi$.

Theorem 3.1. Let $\Phi: E \rightarrow R$ be a $C^{1}$ functional satisfying the Palais Smale condition, and (I1), (I2) and (I3). Further assume there is a constant $\delta>0$ such that

(IS) $\Phi(z) \geq \delta \forall z \in S$,

(IQ) $\Phi(z) \leq 0 \forall z \in \partial Q$.

Then $\Phi$ possesses a critical point with critical value $C \geq \delta$.

The reader is referred to [6] for a proof of this theorem. The critical point given by Theorem 3.1 has a variational characterization we describe next. Let us consider the class of functions

$$
\Gamma=\left\{h \in C(E \times[0,1], E) \mid h \text { satisfies } \Gamma_{1}, \Gamma_{2} \text { and } \Gamma_{3}\right\}
$$

where

$\left(\Gamma_{1}\right) h$ is given by $h(z, t)=\exp (\omega(z, t) L) z+K(z, t)$ where $\omega: E \times$ $[0,1] \rightarrow \mathbb{R}_{0}^{+}$is continuous and transforms bounded sets into bounded sets, and $K: E \times[0,1] \rightarrow E$ is compact.

$\left(\Gamma_{2}\right) h(z, t)=z$

$\forall z \in \partial Q, \forall t \in[0,1]$.

$\left(\Gamma_{3}\right) h(z, 0)=z$

$\forall z \in Q$. 
Then the minimax value

$$
C=\inf _{h \in \Gamma} \sup _{z \in Q} \Phi(h(z, 1))
$$

is the critical value given in Theorem 3.1.

In order to use Theorem 3.1 to find critical points of our functional $\Phi$ we see that it is only left to be proved hypothesis (I3). In fact, the structure of our functional is that given by (I1) and (I2) as we saw in $\S 1$, and the P.S. condition together with the geometric conditions (IS) and (IQ) were proved in Propositions $2.1,2.2$ and 2.3 respectively.

Let us prove (I3). First we define the decomposition of $E$ by taking $X=E^{-}$ and $Y=E^{+}$. The operators $B_{1}$ and $B_{2}$ are defined as $B_{1}, B_{2}: E \rightarrow E$

$$
B_{1}(u, v)=\left(\rho^{\mu-1} u, \rho^{\nu-1} v\right)
$$

and

$$
B_{2}(u, v)=\left(\sigma^{\mu-1} u, \sigma^{\nu-1} v\right),
$$

certainly $B_{1}$ and $B_{2}$ are linear, bounded, invertible operators. Here the constants $\rho$ and $\sigma$ are those of Propositions 2.2 and 2.3 respectively. To show that $\widehat{B}(\omega)$ is invertible we first give a formula for $\exp (\omega L)$.

Lemma 3.1. If $\omega \in \mathbb{R}$ then the operator $\exp (\omega L): E \rightarrow E$ is given by

$$
\exp (\omega L)(u, v)=\cosh (\omega)(u, v)+\sinh (\omega)\left(A^{-s} \circ A^{t} v, A^{-t} \circ A^{s} u\right) .
$$

Proof. We recall from Proposition 1.1 that

$$
L(u, v)=\left(A^{-s} \circ A^{t} v, A^{-t} \circ A^{s} u\right) .
$$

Then $L^{2}=\mathrm{id}_{E}$. By writing explicitly the exponential operator as a series, using (3.10) and reordering the terms we obtain (3.9).

Proposition 3.1. The operator $\widehat{B}(\omega): E^{-} \rightarrow E^{-}$is invertible.

Proof. Given $z \in E^{-}$we have $z=\left(u,-A^{-t} \circ A^{s} u\right)$ with $u \in E^{s}$. By definition of $B_{2}$ in (3.8) we have

$$
B_{2} z=\left(\sigma^{\mu-1} u,-\sigma^{\nu-1} A^{-t} \circ A^{s} u\right),
$$

then using Lemma 3.1 if we write $\exp (\omega L) B_{2} z=(x, y)$ we have

$$
\begin{aligned}
& x=\left(\cosh (\omega) \sigma^{\mu-1}-\sinh (\omega) \sigma^{\nu-1}\right) u, \\
& y=\left(-\cosh (\omega) \sigma^{\nu-1}+\sinh (\omega) \sigma^{\mu-1}\right) A^{-t} \circ A^{s} u .
\end{aligned}
$$

By definition of $B_{1}$ given in (3.7) and from (3.12) and (3.13), if we write $B_{1} \exp (\omega L) B_{2} z=(\bar{x}, \bar{y})$ we have

$$
\begin{aligned}
& \bar{x}=\frac{\cosh (\omega) \sigma^{\mu-1}-\sinh (\omega) \sigma^{\nu-1}}{\rho^{\mu-1}} u, \\
& \bar{y}=\frac{-\cosh (\omega) \sigma^{\nu-1}+\sinh (\omega) \sigma^{\mu-1}}{\rho^{\nu-1}} A^{-t} \circ A^{s} u .
\end{aligned}
$$

Finally we project back into $E^{-}$. If we put $\widehat{B}(\omega) z=(\phi, \psi)$ then using the projection formula given in Lemma 2.1 and (3.14) and (3.15), after some calculations we obtain

$$
\phi=\left\{\frac{1}{2}\left(\frac{\sigma^{\mu-1}}{\rho^{\mu-1}}+\frac{\sigma^{\nu-1}}{\rho^{\nu-1}}\right) \cosh (\omega)-\frac{1}{2}\left(\frac{\sigma^{\nu-1}}{\rho^{\mu-1}}+\frac{\sigma^{\mu-1}}{\rho^{\nu-1}}\right) \sinh (\omega)\right\} u
$$


If we put $m$ as the coefficient of $u$ in (3.16) we also find that

$$
\psi=-m A^{-t} \circ A^{s} u \text {. }
$$

In other words we have that $\widehat{B}(\omega) z=m z$. This constant $m$ is positive if we assume, without loss of generality that $\sigma>1$ and $\rho<1$. In fact

$$
\left(\frac{\sigma^{\nu-1}}{\rho^{\nu-1}}+\frac{\sigma^{\mu-1}}{\rho^{\mu-1}}\right)-\left(\frac{\sigma^{\mu-1}}{\rho^{\nu-1}}+\frac{\sigma^{\nu-1}}{\rho^{\mu-1}}\right)=\frac{\left(\rho^{\mu-1}-\rho^{\nu-1}\right)\left(\sigma^{\nu-1}-\sigma^{\mu-1}\right)}{\rho^{\mu+\nu-2}}
$$

is positive so that $m>0$ independently of the value of $\omega \in \mathbb{R}$. This in turn implies that $\widehat{B}(\omega)$ is invertible.

\section{Proof of the theorems}

Now we are in a position of giving a proof of the theorems announced in the Introduction. The application of Theorem 3.1 will give existence of a critical point of the functional $\Phi$ and then by applying regularity results we obtain a solution of (ES).

Proof of Theorems 0.1 and 0.2 . We apply minimax Theorem 3.1 to the functional $\Phi$. Hypothesis (I1) and (I2) are satisfied by the considerations made in $\S 1$. Definitions (3.7) and (3.8) and Proposition 3.1 give (I3). The Palais Smale condition is proved in Proposition 2.1 and the geometric conditions leading to (IS) and (IQ) are proved in Propositions 2.2 and 2.3 respectively.

Thus, there exists $z \in E$ such that $\Phi^{\prime}(z)=0$ i.e. $z$ is an $(s, t)$-weak solution of (ES). Next, Theorem 2.2 gives that $z=(u, v)$ is such that $u \in$ $W^{2, p /(p-1)}(\Omega) \cap W_{0}^{1, p /(p-1)}(\Omega)$ and $v \in W^{2, q /(q-1)}(\Omega) \cap W_{0}^{1, q /(q-1)}(\Omega)$. That is, $(u, v)$ is a strong solution of (ES).

When the Hamiltonian is independent of $x$ we have that $(0,0)$ is a solution of (ES). Since $\Phi(z) \geq \delta>0$ and $\Phi((0,0))=0$ we find that $z$ is not trivial.

In order to prove Theorem 0.3 we need to redefine the Hamiltonian. Let us define $\widetilde{H}: \mathbb{R}^{2} \times \bar{\Omega} \rightarrow \mathbb{R}$ so that

$$
\widetilde{H}(u, v, x)= \begin{cases}H(u, v, x) & \text { if } u \geq 0, v \geq 0 \\ H(0, v, x) & \text { if } u \leq 0, v \geq 0 \\ H(u, 0, x) & \text { if } u \geq 0, v \leq 0 \\ 0 & \text { if } u \leq 0, v \leq 0\end{cases}
$$

Since we are assuming (H6) the new Hamiltonian $\widetilde{H}$ is of class $C^{1, \varepsilon}$ as $H$. It also satisfies $(\mathrm{H} 1),(\mathrm{H} 3)$ and $(\mathrm{H} 4)$. However $(\mathrm{H} 2)$ is satisfied in a restricted form. Next we modify the arguments given before to include this new situation.

Proof of Theorem 0.3 . First we define the functional $\tilde{\Phi}: E \rightarrow \mathbb{R}$ as

$$
\widetilde{\Phi}(z)=Q(z)-\int_{\Omega} \widetilde{H}(z, x) d x .
$$

It is clear that $\widetilde{\Phi}$ is of class $C^{1}$ in $E$. Assume there exists $z=(u, v)$ a critical 
point of $\widetilde{\Phi}$. Then by the argument given before we have a classical solution of

$$
\begin{gathered}
-\Delta u=\frac{\partial \widetilde{H}}{\partial v}(u, v, x) \text { in } \Omega, \\
-\Delta v=\frac{\partial \widetilde{H}}{\partial u}(u, v, x) \text { in } \Omega, \\
u=0, \quad v=0 \text { on } \partial \Omega .
\end{gathered}
$$

Since $\partial \widetilde{H} / \partial u \geq 0$ and $\partial \widetilde{H} / \partial v \geq 0$ by the maximum principle we find that $u>0$ and $v>0$ in $\Omega$. We only need to prove that hypotheses of Theorem 3.1 still hold. Hypothesis (H2) was used only in proving the Palais Smale condition and the geometric condition (IQ).

Let us see first how we prove the Palais condition for $\widetilde{\Phi}$. Let $\left\{z_{n}\right\}$ be a sequence in $E$ so that

$$
\left|\widetilde{\Phi}\left(z_{n}\right)\right| \leq c \text { and } \widetilde{\Phi}^{\prime}\left(z_{n}\right) \rightarrow 0, \quad \text { as } n \rightarrow \infty .
$$

As we did in Proposition 2.1 we have only to prove that (4.4) implies that $\left\{z_{n}\right\}$ is bounded. Proceeding as in Proposition 2.1 we find

$$
c_{1}\left(1+\left\|z_{n}\right\|_{E}\right) \geq \int_{\Omega} \tilde{H}\left(u_{n}, v_{n}, x\right) d x
$$

where we used the hypothesis $(\mathrm{H} 2)$ restricted to $u \geq 0$ and $v \geq 0$. Then, after Remark 0.5 we find $c_{2}$ so that

$$
\int_{\Omega}\left|u_{n}^{+}\right|^{\alpha}+\left|v_{n}^{+}\right|^{\beta} d x \leq c_{2}\left(1+\left\|u_{n}\right\|_{E^{s}}+\left\|v_{n}\right\|_{E^{t}}\right) .
$$

Where we denote by $u_{n}^{+}$and $v_{n}^{+}$the positive part of $u_{n}$ and $v_{n}$ respectively. Next, following the proof of Proposition 2.1 we obtain

$$
\left\|v_{n}\right\|_{E^{t}} \leq c_{4}\left(\left\|u_{n}^{+}\right\|_{L^{\alpha}(\Omega)}^{p-1}+\left\|v_{n}^{+}\right\|_{L^{\beta}(\Omega)}^{(p-1) q / p}+1\right) .
$$

By an analogous reasoning

$$
\left\|u_{n}\right\|_{E^{s}} \leq c_{5}\left(\left\|v_{n}^{+}\right\|_{L^{\beta}(\Omega)}^{q-1}+\left\|u_{n}^{+}\right\|_{L^{\alpha}(\Omega)}^{(q-1) p / q}+1\right) .
$$

Substituting (4.7) and (4.8) into (4.6) we obtain

$$
\begin{aligned}
& \left\|u_{n}^{+}\right\|_{L^{\alpha}(\Omega)}^{\alpha}+\left\|v_{n}^{+}\right\|_{L^{\beta}(\Omega)}^{\beta} \\
& \quad \leq c_{6}\left(\left\|u_{n}^{+}\right\|_{L^{\alpha}(\Omega)}^{p-1}+\left\|v_{n}^{+}\right\|_{L^{\beta}(\Omega)}^{(p-1) q / p}+\left\|u_{n}^{+}\right\|_{L^{\alpha}(\Omega)}^{(q-1) p / q}+\left\|v_{n}^{+}\right\|_{L^{\beta}(\Omega)}^{q-1}+1\right) .
\end{aligned}
$$

By our assumption (i) and (iii) on the exponents $\alpha, \beta, p$ and $q$ we find that $\left\|u_{n}^{+}\right\|_{L^{\alpha}(\Omega)}$ and $\left\|v_{n}^{+}\right\|_{L^{\beta}(\Omega)}$ are bounded. Next we can apply (4.7) and (4.8) again to obtain that $\left\{z_{n}\right\}$ is bounded in $E$. This ends the proof of P.S. condition.

Now we consider (IQ). Keeping the definitions of $Q$ given in $\S 2$ we take the function $z_{+}=\left(u_{+}, v_{+}\right) \in E^{+}$so that $u_{+}=\phi_{1}$ and $v_{+}=A^{-t} A^{s} \phi_{1}=\lambda_{1}^{-t+s} \phi_{1}$, where $\phi_{1}$ is the first eigenfunction of $-\Delta$. In particular $\phi_{1}>0$ in $\Omega$. We need to prove the existence of the constants $\sigma$ and $M$ used in the definition of $Q$.

Since we have

$$
\left(\tau \phi_{1}+u\right)^{+}=\left((\tau+\gamma) \phi_{1}+\hat{u}\right)^{+} \geq(\tau+\gamma) \phi_{1}+\hat{u},
$$


we proceed as in the estimate (2.28) and get

$$
\tau+\gamma \leq c\left\|\left(\tau u_{+}+u\right)^{+}\right\|_{L^{\alpha}(\Omega)} .
$$

Similarly we obtain

$$
\tau-\gamma \leq c\left\|\left(\tau v_{+}+v\right)^{+}\right\|_{L^{\beta}(\Omega)} .
$$

From here on we proceed as in Proposition 2.3.

Note added in proof. After this paper was finished we learned from Hulshof and van der Vorst that they have obtained similar results in their paper Differential systems with strongly indefinite variational structure, J. Funct. Anal. 114 (1993), 32-58.

\section{REFERENCES}

1. R. A. Adams, Sobolev spaces, Academic Press, 1975.

2. V. Benci and P. Rabinowitz, Critical point theorems for indefinite functionals, Invent. Math. 52 (1979), 241-273.

3. D. G. Costa and C. A. Magalhães, $A$ variational approach to subquadratic perturbations of elliptic systems, Preprint.

4. Ph. Clément, D. G. de Figueiredo and E. Mitidieri, Positive solutions of semilinear elliptic systems, Comm. Partial Differential Equations 17 (1992), 923-940.

5. __ Estimates of positive solutions of systems via Hardy-Sobolev inequalities, Preprint.

6. P. Felmer, Periodic solutions of 'superquadratic' Hamiltonian systems, J. Differential Equations 102 (1993), 188-207.

7. D. Fujiwara, Concrete characterization of the domains of fractional powers of some elliptic differential operators of the second order, Proc. Japan Acad. 43 (1967).

8. B. Gidas and J. Spruck, A priori bounds for positive solutions of nonlinear elliptic equations, Comm. Partial Differential Equations 6 (1981), 883-901.

9. D. Gilbarg and N. S. Trudinger, Elliptic partial differential equations of second order, Springer-Verlag, Berlin, 1983.

10. J. L. Lions and E. Magenes, Problèmes aux limites non homogènes et applications, Vol. I, Dunod, Paris, 1968.

11. A. Persson, Compact linear mappings between interpolation spaces, Ark. Mat. 5 (1964), 215-219.

12. P. Rabinowitz, Minimax methods in critical point theory with applications to differential equations, CBMS Regional Conf. Ser. in Math., no. 65, Amer. Math. Soc., Providence, R.I., 1986.

13. M. A. Souto, Ph.D. Thesis, UNICAMP, Brasil, 1992.

IMECC-UNICAMP, Caixa Postal 6065, 13081 Campinas S. P. Brazil

E-mail address: djairo@ime.unicamp.br

Departamento de Ingenieria Matemática, F.C.F.M., Universidad de Chile, Casilla 170 Correo 3, Santiago, Chile

E-mail address: pfelmerQuchcecrm.bitnet 